\title{
Relación entre el Bienestar Hedónico y Bienestar Eudaimónico en pacientes pediátricos oncológicos ${ }^{5}$
}

\author{
Aura Pérez Mendoza \\ Psicóloga \\ Universidad del Sinú, Montería, Colombia \\ Correo electrónico: aucripeme16@hotmail.com \\ Stefano Vinaccia Alpi \\ Doctor en psicología clínica y de la salud \\ Universidad del Sinú, Montería, Colombia \\ Correo electrónico: vinalpi47@hotmail.com
}

Rosangela Raleigh Bethencourt

Psicóloga

Universidad del Sinú, Montería, Colombia Correo electrónico: rosangela.raleigh@gmail.com

Recibido: 29/11/2018

Evaluado: $15 / 01 / 2019$

Aceptado: 31/01/2019

\section{Resumen}

El propósito de este estudio fue determinar la relación entre el Bienestar Hedónico y Bienestar Eudaimónico en pacientes oncológicos infanto-juveniles en Córdoba, Colombia. La investigación fue de corte transversal, de alcance descriptivo-correlacional. Se aplicaron los cuestionarios de Satisfacción con la Vida de Diener para medir Bienestar Hedónico y de Bienestar Psicológico de Ryff para Bienestar Eudaimónico a una muestra de 30 pacientes con diagnóstico de cáncer en la ciudad de Montería. Los resultados arrojaron niveles óptimos de Bienestar Eudaimónico y Hedónico en los pacientes. Las dos variables presentaron, en general, correlaciones bajas con excepción de la dimensión de Autoaceptación de la escala de Ryff que presentó una correlación moderada y positiva con Satisfacción con la vida. En conclusión, se hace necesario continuar investigando otras variables que pueden moderar esta relación.

Bienestar Hedónico, Bienestar Eudaimónico, Adolescentes, niños, cáncer.

5 Para citar este artículo: Perez, A.C., Vinaccia, S. \& Raleigh, R. (2020). Relación entre el Bienestar Hedónico y Bienestar Eudaimónico en pacientes pediátricos oncológicos. Informes Psicológicos, 20(1), pp. 63-74 http://dx.doi. org/10.18566/infpsic.v20n1a05 


\title{
Relationship between hedonic and eudaimonic well-being in pediatric cancer patients
}

\begin{abstract}
The purpose of this study was to determine the relationship between hedonic and eudaimonic well-being in childhood and juvenile cancer patients in Córdoba, Colombia. The research was cross-sectional, descriptive-correlational. Diener's Satisfaction with Life questionnaires were applied to measure Ryff's hedonic well-being and psychological well-being for eudaimonic well-being to a sample of 30 patients diagnosed with cancer in the city of Montería. The results showed optimal levels of eudaimonic and hedonic well-being in the patients. In general, the two variables presented low correlations with the exception of the Self-acceptance dimension of the Ryff scale, which presented a moderate and positive correlation with Satisfaction with life. In conclusion, it is necessary to conduct further research on other variables that can moderate this relationship.
\end{abstract}

Keywords Hedonic well-being, eudaimonic well-being, adolescents, children, cancer.

\section{Relação entre o bem-estar hedónico e o bem-estar eudaimónico em pacientes pediátricos oncológicos}

\section{Resumo}

0 propósito deste estudo foi determinar a relação entre o bem-estar hedónico e 0 bem-estar eudaimónico em pacientes oncológicos na idade infantil e juvenil em Córdoba, Colômbia. A pesquisa foi transversal, de alcance descritivo-correlacional. Foram implementados os questionários de satisfação com a vida de Diener para a medição do bem-estar hedónico, e do bem-estar psicológico de Ryff para o bem-estar eudamónico a uma amostra de 30 pacientes com câncer diagnosticado na cidade de Montería. Os resultados exibiram níveis ótimos de bem-estar eudaimónico e hedónico nos pacientes. As duas variáveis apresentaram, de maneira geral, correlações baixas com exceção da dimensão de auto aceitação da escala de Ryff que apresentou uma correlação moderada e positiva com satisfação com a vida. Em conclusão, torna-se preciso continuar pesquisando outras variáveis que possam moderar esta relação. 


\section{ntroducción}

El cáncer se constituye como una enfermedad que amenaza el bienestar de la población y supone un riesgo vital, con tendencia a tener mayor impacto a nivel mundial tanto en adultos como en niños en los siguientes años. Según estudios realizados por la Organización Mundial de la Salud (OMS), se registraron 8.8 millones de defunciones relacionadas con cáncer en 2015, colocando a esta enfermedad dentro de las primeras causas de muerte a nivel global. En relación al cáncer infantil, tiene una incidencia entre 50 y 200 por cada millón de niños (OMS, 2016).

En Colombia, según el Ministerio de Salud y Protección Social, 33 mil personas al año mueren de cáncer, dentro de las cuales 16.800 son mujeres y 16.300 son hombres. En la población infantil, se estima que en el país se diagnostican anualmente alrededor de 2.200 casos nuevos. Sin embargo, solo el 50\% logra superarlo; mientras que, en países más desarrollados, esta cifra aumenta a 80\% (Ministerio de Salud, 2011). Se estima que entre 2005 y 2011 se presentaron 219 muertes por cáncer infantil al año en el departamento colombiano de Córdoba, siendo el octavo con mayores cifras ( $\mathrm{Mi}$ nisterio de Salud, 2013).

En el cáncer, el proceso médico y mejoramiento de los pacientes en el campo físico es un objetivo, al igual que la búsqueda de su bienestar, dado que el área emocional, social y escolar también se ven afectadas en el diagnóstico de la enfermedad (Pérez, 2015). El niño pasa de tener un rol social activo-dependiente acompañado de juegos, a un rol totalmente pasivo-dependiente caracterizado por la inactividad, debido a las limitaciones propias de la condición en la que se encuentra, lo cual causa una interferencia en su bienestar psicológico y calidad de vida (Nearchou, Campbell, Duffy, Fehily \& Ryan, 2017).

El estudio del bienestar parte de dos tradiciones parcialmente solapadas que se desarrollan en paralelo, buscando la explicación de felicidad basándose en diferentes soportes filosóficos. Por un lado, el bienestar subjetivo o hedónico tiene relación con la satisfacción vital del placer y la afectividad, donde brevemente se explica que, cuanto menos dolor, mayor placer y sensación de felicidad se presenta (Proctor, 2014). Por otro lado, el bienestar psicológico se relaciona con la tradición eudaimonista, la cual establece "un estado deseable al que sólo se puede aspirar llevando una vida virtuosa o deseable, calificada como tal desde un particular sistema de valores" (García, 2002, p. 21). Sin embargo, en un relevante trabajo sobre la distinción entre felicidad y eudaimonia de Telfer (1980), se establece que la satisfacción con la vida, el indicador más empleado de bienestar subjetivo, incluye componentes claramente eudaimónicos.

Investigaciones en adolescentes han demostrado que el bienestar depende de factores, tanto internos como externos, principalmente relacionados con estatus socioeconómico, satisfacción con la imagen corporal y autoconcepto. En otras palabras, los grandes cambios físicos, sociales y cognitivos que se experimentan durante este período evolutivo tienen una gran influencia sobre el ajuste social y psicológico, así como en las conductas de riesgo que ejercen los adolescentes (Orcasita, Mosquera, \& Carrillo, 2018; 
Žukauskienè, 2013). Estas dificultades se suman a los efectos negativos sobre bienestar en adolescentes que sufren enfermedades crónicas, particularmente, el cáncer (Balázs et al., 2018; Warner et al., 2016). Cabe destacar que no se han encontrado investigaciones que estudien la relación entre bienestar hedónico y eudaimónico dentro de esta población.

Al determinar la relación entre el bienestar hedónico y eudaimónico en pacientes infanto-juveniles, se puede iniciar una recolección de datos que permitan contribuir con la promoción del bienestar en el paciente y prevenir trastornos en sus campos de acción social y emocional; por lo que el objetivo de esta investigación se centró en determinar la relación entre el Bienestar Hedónico y Bienestar Eudaimónico en pacientes oncológicos infanto-juveniles en Córdoba, Colombia.

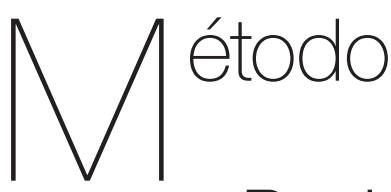

\section{Participantes y muestreo}

La muestra estuvo conformada por 30 niños y adolescentes con cáncer del departamento de Córdoba, Colombia entre 11 a 18 años ( $M=15.27$; $D E=1.70)$, escogidos mediante un muestreo de tipo no aleatorio de sujetos disponibles. Se encontraban en tratamiento farmacológico y el tiempo de diagnóstico oscilaba entre 6 y 48 meses (ver Tabla 1).

El muestreo fue no aleatorio de tipo intencional por sujetos disponibles, que asistían a la unidad de Oncohematología del Instituto Médico de Alta Tecnología (IMAT) de Montería, Córdoba.

\section{Instrumentos}

Escala de Bienestar Psicológico de Ryff (Ryff, 1995). Su revisión fue realizada por Dirk van Dierendonck (2004); adaptada al español por Díaz et al. (2006) y fue validada únicamente para adultos jóvenes colombianos por Pineda, Castro y Chaparro (2018), presentando un índice de confiabilidad de .91.

Es una prueba que mide el Bienestar Eudaimónico percibido dividido en seis dimensiones: dominio del entorno, crecimiento personal, propósito en la vida, autonomía, relaciones positivas con otros y autoaceptación. Se utilizó la versión traducida de 29 ítems con seis opciones de respuesta que van de totalmente de acuerdo a totalmente en desacuerdo.

Escala de satisfacción con la vida SWLS (Diener, 2006). La validación española de esta escala fue realizada por Vázquez, Duque y Hervás (2013), mientras que la validación para jóvenes colombianos fue realizada por Vinaccia, Riveros, Vera, Quiceno, y Parra (en prensa), donde la escala obtuvo un alfa de Cronbach de .839.

Esta escala mide Bienestar Hedónico y se encuentra constituida por cinco preguntas, donde se evalúan utilizando una puntuación entre 1 y 7 , siendo 1 completamente en desacuerdo y 7 completamente de acuerdo. La puntuación máxima posible es, por tanto, 35.

\section{Procedimiento}

Se seleccionó la muestra a evaluar en la investigación según el diagnóstico y la edad de los participantes. Una vez aprobado el proyecto por el comité ético de la 
Universidad del Sinú y el Instituto Medico de Alta Tecnología (IMAT), la recolección de los datos se realizó de manera directa e individual en un período de dos meses, procediendo antes a invitar a las madres o familiar a cargo para acudir con sus hijos a un lugar previamente designado donde se les solicitó el consentimiento informado de la madre (o familiar) y el asentimiento del niño para la participación en el estudio.

En este apartado del consentimiento informado se aclararon los pasos que se realizarían en la investigación, explicando la aplicación de la escala de Ryff y la Satisfacción con la Vida o SWLS (Satisfaction With Life Scale), tomando en cuenta las normativas de la Declaración Universal de Principios Éticos para Psicólogos (International Union of Psychological Science [IUPS], 2008) y el Código de Conducta y Principios Éticos para Psicólogos de la Asociación Americana de Psicología (APA, 2017) para preservar la dignidad de los participantes de la investigación. Se procedió a la creación de la base de datos en Microsoft Excel y análisis en el paquete estadístico SPSS v24, donde se obtuvieron medidas de tendencia central y correlaciones.

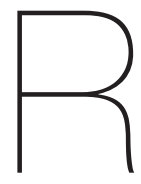

\section{esultados}

Se analizaron de manera descriptiva las variables sociodemográficas, la satisfacción con la vida y el bienestar psicológico; para esto se calcularon porcentajes y medidas de tendencia central. Para el análisis correlacional, se utilizó el coeficiente rho de Spearman para las variables satisfacción con la vida y bienestar subjetivo, debido a que, de acuerdo a los resultados obtenidos en la prueba de normalidad KolmogorovSmirnov, se concluyó que las variables no obedecían a una distribución normal.

Quienes conformaban la muestra fueron jóvenes con una edad promedio de 15.27 años, en su mayoría de género femenino (63.33\%), cursantes de secundaria, de estrato socioeconómico bajo y adscritos al sistema de salud EPS. El tipo de cáncer más común dentro de la muestra fue la leucemia, seguido por los tumores sólidos. Todos se encontraban en tratamiento al momento de la aplicación de la prueba y tenían en promedio 18.73 meses de diagnóstico.

Tabla 1.

Datos sociodemográficos

\begin{tabular}{|c|c|c|c|c|c|}
\hline Sexo & $\mathrm{N}$ & $\%$ & $\begin{array}{l}\text { Sistema de } \\
\text { salud al cual } \\
\text { está adscrito }\end{array}$ & $\mathrm{N}$ & $\%$ \\
\hline Mujeres & 19 & 63.33 & EPS & 28 & 93.33 \\
\hline Hombres & 11 & 36.67 & Particular/privada & 2 & 6.67 \\
\hline Nivel de estudio & & & Tipo de cáncer & & \\
\hline Primaria $\left(1^{\circ} \mathrm{a} 5^{\circ}\right)$ & 8 & 26.67 & Tumor sólido & 10 & 33.33 \\
\hline $\begin{array}{l}\text { Secundaria } \\
\left(6^{\circ} \text { a } 9^{\circ}\right)\end{array}$ & 15 & 50 & Leucemia & 17 & 56.67 \\
\hline Media $\left(10^{\circ}\right.$ a $\left.11^{\circ}\right)$ & 7 & 23.33 & $\begin{array}{l}\text { Linfoma hodgkin } \\
\text { /No Hodgkin }\end{array}$ & 3 & 10 \\
\hline Técnico, tecnólogo & 0 & 0 & & & \\
\hline $\begin{array}{l}\text { Estrato } \\
\text { socioeconómico }\end{array}$ & & & & M & $\mathrm{DE}$ \\
\hline 1 & 21 & 70 & Edad & 15.27 & 1.70 \\
\hline 2 & 7 & 23.33 & $\begin{array}{l}\text { Tiempo (Meses) } \\
\text { de diagnóstico }\end{array}$ & 18.73 & 11.83 \\
\hline 3 & 1 & 3.33 & & & \\
\hline 4 & 1 & 3.33 & & & \\
\hline
\end{tabular}


El puntaje que representa la media de bienestar psicológico en los participantes oncológicos fue de 29.64 puntos, con una desviación de 7.60. Se considera que, en general, la muestra presentó de adecuados niveles de Bienestar Psicológico. En cuanto a la Satisfacción con la Vida, el punto de corte para identificar bajos niveles de satisfacción con la vida es por debajo de 20, por lo que, al obtener una media de 28.97 puntos, se puede afirmar que se obtuvo una elevada satisfacción en los participantes (Pavot \& Diener, 2008).

Tabla 2.

Resultado de Bienestar Psicológico en pediátricos y adolescentes oncológicos.

\begin{tabular}{lccccc}
\hline & Media & D.T & Min. & Máx. & $\alpha$ \\
\hline $\begin{array}{l}\text { Escala de Satisfac- } \\
\text { ción con la vida }\end{array}$ & 28.97 & 7.60 & 10 & 35 & .949 \\
Autoaceptación & 5.35 & .78 & 2.5 & 6 & .624 \\
Relaciones positivas & 4.75 & 1.01 & 2.6 & 6 & .585 \\
Autonomía & 4.8 & 1.12 & 2.33 & 6 & .770 \\
Dominio del entorno & 4.43 & .89 & 2.6 & 5,8 & .121 \\
Propósito de vida & 5.31 & .81 & 2 & 6 & .736 \\
Crecimiento personal & 5.08 & .89 & 2.75 & 6 & .625 \\
\hline
\end{tabular}

Se clasificaron los puntajes obtenidos en Bienestar Psicológico y Satisfacción con la Vida por la muestra en bajos, medios y altos; tomando en cuenta la desviación estándar y la media. En la Tabla 3 , se puede apreciar esta clasificación en relación al tipo de tumor que presentaron los pacientes. En su mayoría, los participantes reportaron valores medios a altos en Bienestar Subjetivo y Satisfacción con la vida.
Tabla 3.

Valores de Bienestar Eudaimónico y Hedónico con respecto al tipo de cáncer.

\begin{tabular}{|c|c|c|c|c|c|}
\hline & \multicolumn{3}{|c|}{ Tipo de tumor } & \multirow[b]{2}{*}{ Total } \\
\hline & & $\begin{array}{l}\text { Tumor } \\
\text { sólido }\end{array}$ & Leucemia & $\begin{array}{c}\text { Linfoma } \\
\text { Hodgkin/ } \\
\text { No Hodgkin }\end{array}$ & \\
\hline \multirow{2}{*}{ Autoaceptación } & Bajo & 2 & 2 & 0 & 4 \\
\hline & Medio-Alto & 8 & 15 & 3 & 26 \\
\hline Total & & 10 & 17 & 3 & 30 \\
\hline \multirow{3}{*}{$\begin{array}{l}\text { Relaciones } \\
\text { positivas }\end{array}$} & Bajo & 2 & 4 & 0 & 6 \\
\hline & Medio & 4 & 10 & 3 & 17 \\
\hline & Alto & 4 & 3 & 0 & 7 \\
\hline \multirow[t]{2}{*}{ Total } & & 10 & 17 & 3 & 30 \\
\hline & Bajo & 3 & 3 & 0 & 6 \\
\hline \multirow[t]{2}{*}{ Autonomía } & Medio & 7 & 11 & 3 & 21 \\
\hline & Alto & 0 & 3 & 0 & 3 \\
\hline \multirow[t]{2}{*}{ Total } & & 10 & 17 & 3 & 30 \\
\hline & Bajo & 3 & 1 & 0 & 4 \\
\hline \multirow[t]{2}{*}{$\begin{array}{l}\text { Dominio del } \\
\text { entorno }\end{array}$} & Medio & 6 & 13 & 3 & 22 \\
\hline & Alto & 1 & 3 & 0 & 4 \\
\hline Total & & 10 & 17 & 3 & 30 \\
\hline \multirow{2}{*}{$\begin{array}{l}\text { Propósito } \\
\text { de vida }\end{array}$} & Bajo & 0 & 3 & 0 & 3 \\
\hline & Medio-Alto & 10 & 14 & 3 & 27 \\
\hline \multirow[t]{2}{*}{ Total } & & 10 & 17 & 3 & 30 \\
\hline & Bajo & 1 & 2 & 0 & 3 \\
\hline \multirow[t]{2}{*}{$\begin{array}{l}\text { Crecimiento } \\
\text { personal }\end{array}$} & Medio & 6 & 9 & 2 & 17 \\
\hline & Alto & 3 & 6 & 1 & 10 \\
\hline Total & & 10 & 17 & 3 & 30 \\
\hline \multirow{2}{*}{$\begin{array}{l}\text { Satisfacción } \\
\text { con la vida }\end{array}$} & Bajo & 1 & 4 & 0 & 5 \\
\hline & Medio-Alto & 9 & 13 & 3 & 25 \\
\hline Total & & 10 & 17 & 3 & 30 \\
\hline
\end{tabular}

Se identificó que existen bajas correlaciones entre las variables Satisfacción con la vida y las dimensiones de Bienestar Psicológico, a excepción de una relación significativa al nivel .05, moderada y positiva entre Satisfacción con la vida y la dimensión de Autoaceptación ( $\left.r=.442^{*}\right)$, la cual se puede apreciar en la Tabla 4. 
Tabla 4.

Análisis de correlación entre bienestar psicológico y satisfacción con la vida.

\begin{tabular}{|c|c|c|c|c|c|c|c|}
\hline & $\begin{array}{l}\text { Bienestar } \\
\text { psicológico } \\
\text { total }\end{array}$ & $\begin{array}{c}\text { Auto } \\
\text { aceptación }\end{array}$ & $\begin{array}{c}\text { Relaciones } \\
\text { positivas }\end{array}$ & Autonomía & $\begin{array}{c}\text { Dominio } \\
\text { del } \\
\text { entorno }\end{array}$ & $\begin{array}{c}\text { Crecimiento } \\
\text { personal }\end{array}$ & $\begin{array}{c}\text { Propósito } \\
\text { de vida }\end{array}$ \\
\hline $\begin{array}{l}\text { Satisfacción } \\
\text { con la vida }\end{array}$ & .333 & $.442^{\star}$ & .208 & 197 & .200 & 196 & .225 \\
\hline
\end{tabular}

Nota: **. La correlación es significativa en el nivel 0.01 (bilateral); *. La correlación es significativa en el nivel .05 (bilateral).

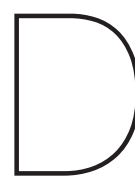

\section{iscusión}

En primer lugar, las puntuaciones elevadas de la Escala de Bienestar de Ryff evidencian que más del $80 \%$ de los participantes tienen un nivel de BP entre moderado y alto, y que solo el $13 \%$ de los participantes posee bajos métodos de proyección o afrontamiento de los retos, es decir, tienen dificultad en la forma de manejar y controlar las situaciones para aprender de ellos a futuro.

Un estudio realizado sobre el Bienestar Psicológico y subjetivo, y su relación con la salud en personas mayores y jóvenes por Concepción (2015), denotó que, a mayor grado de salud en la persona, la escala de Bienestar Psicológico de Ryff declarará una mayor puntuación. En otro estudio, Novoa, Caycedo, Aguillón y Suárez (2008) también aseveran que el bienestar psicológico en pacientes oncológicos, en este caso adultos, se ve claramente afectado; de igual manera, se han observado conexiones entre la enfermedad médica y morbilidad psicológica (Valencia, 2006). Este conjunto de hallazgos se contradice al compararlos con los resultados reflejados en esta investigación, ya que se encontraron niveles altos en BP y BS, de acuerdo a la clasificación de los resultados realizada, aun cuando la muestra se encuentra en una etapa cronológica de grandes cambios, son pacientes oncológicos y, además, pertenecen a un estrato socioeconómico bajo.

Sin embargo, es posible que grupos que se identifican como 'vulnerables', puedan presentar niveles altos de Bienestar Subjetivo, e incluso, condiciones como ser pertenecientes a un grupo étnico minoritario son predictores de bienestar subjetivo, contrario a lo que se espera debido a los constantes retos a los que son expuestos estos grupos (Ryff, 2017). De igual manera, en población de mujeres con cáncer de mama, también se han podido encontrar altos niveles de bienestar subjetivo (Deledda et al., 2015). Estos autores estudiaron, además, otras variables como aceptación y flexibilidad psicológica, las cuales podrían moderar la presencia de bienestar subjetivo.

Las dimensiones de Autoaceptación, Propósito de vida y Crecimiento personal fueron las dimensiones con puntuaciones más altas, seguidas por Autonomía, Relaciones positivas y Dominio del entorno. En búsqueda de bases teóricas que expliquen las tres puntuaciones bajas en comparación con Autoaceptación, Propósito 
de Vida y Crecimiento Personal, se expone una congruencia en investigaciones previas por Fernández, Muratori y Zubieta (2013), donde se apreció una correlación directa con el aumento de la edad y las tres dimensiones del bienestar psicológico: Autonomía, Dominio del entorno y Relaciones positivas, puesto que se encontró mayor sentido a las experiencias vividas y una mayor capacidad para manejar el contexto social, a fin de satisfacer las necesidades y valores a medida que aumenta la edad. En pocas palabras, cuanta menor edad, menor control de autonomía, dominio del entorno y relaciones positivas.

En cuanto a la Satisfacción con la Vida (o Bienestar Hedónico), presentó un alto puntaje de la escala de satisfacción en los pediátricos oncológicos. Al igual que en $\mathrm{BP}$, se encontró que más del $80 \%$ de la muestra reporta satisfacción con la vida y que el $13 \%$ de los participantes tienen valores bajos o deficientes en satisfacción vital, es decir, problemas en la capacidad de generar creencias positivas y satisfacción frente a experiencias diarias. Investigaciones previas realizadas por Mella et al. (2004) y Meléndez, Tomás y Navarro (2008) coinciden con estos resultados, manifestando que, a medida que aumenta la edad, la satisfacción vital disminuye, por lo que se considera que los menores tienden a una mayor satisfacción con la vida que los adultos. Esto es concordante con que el bienestar varía de acuerdo a la edad (Keyes, Shmotkin, \& Ryff, 2002), siendo los adultos los que evalúan más negativamente su vida (Liberalesso, 2002). Otras investigaciones contradicen los resultados expresando una relación directa entre la satisfacción vital y la salud (Concepción, 2015); sin embargo, en este caso no parece haber una afectación del bienestar debido a la enfermedad.

Por otro lado, analizando la correlación entre Bienestar Psicológico (BP) o Eudaimónico y de Bienestar Subjetivo (BS) o Hedónico medido a través de los resultados en Satisfacción con la vida, fueron bajas, a excepción de la relación significativa, moderada y positiva entre BS y Autoaceptación, una de las dimensiones pertenecientes a BP. Estos datos muestran una estructura de dos componentes relacionados, corroborados en estudios previos por Reina, Oliva y Parra (2010); Burgos (2012) y Muratori y Zubieta (2015), donde se especifica que la felicidad subjetiva se armoniza directamente y con mayor fuerza a la dimensión de Autoaceptación, puesto que la necesidad de creencias positivas al momento de satisfacerlas genera en el individuo alta autoestima y aceptación de sí mismo. Se ha comprobado que las autoevaluaciones cumplen un rol fundamental en el ajuste y en la calidad de vida de los adolescentes: el concepto que uno tiene de sí mismo predice la satisfacción con la vida y el bienestar subjetivo (McCullough, Huebner \& Laughlin, 2000).

A raíz de múltiples estudios, ya se había establecido una relación moderada entre las dimensiones Autoaceptación y Dominio del entorno y el BS, a diferencia del resto de las dimensiones del BP, las cuales mostraron relaciones débiles (Keyes et al., 2002), repitiéndose dicha relación de interdependencia, siendo constructos distintos, en la muestra de esta investigación.

componente de Autoaceptación contribuye en los aspectos y creencias positivas a la hora de determinar si una 
persona se siente feliz consigo mismo y su alrededor. Estos datos permiten establecer los aspectos beneficiosos que maneja el BP, teniendo en cuenta que los participantes son menores de edad, perteneciente la mayoría a estratos socioeconómicos bajos y con enfermedades crónicas del departamento de Córdoba.

En general, se puede concluir que la muestra de niños y adolescentes con cáncer estudiada presenta altos niveles de Satisfacción con la Vida (hedonismo), así como de esfuerzo en proyectarse y ejecutar planes o metas personales para su crecimiento personal (eudaimonismo), a pesar de encontrarse en un estado de salud precario y en tratamientos o control médico debido a su patología. Esto puede deberse a la influencia de múltiples variables, tanto psicológicas como de índole social, que median el estado de bienestar tanto subjetivo como psicológico, por lo que se hace necesario continuar con el estudio de variables como flexibilidad psicológica y aceptación, al igual que apoyo social y resiliencia; ya que han sido estudiantes en población similar y parecen ser relevantes.

Dentro de las limitaciones, se puede mencionar que únicamente se trabajó con niños y adolescentes que eran atendidos en el instituto médico al momento de la aplicación. Cabe destacar, además, que los instrumentos utilizados durante este estudio se encontraban únicamente validados para población adulta colombiana, es decir, las conclusiones de este estudio son pertinentes solo para la población estudiada, ya que no se pueden generalizar a otros pacientes colombianos y los instrumentos no se encuentran validados para la población pediátrica y juvenil. Para futuras investigaciones, se recomienda replicar en otras poblaciones dentro del país y profundizar metodológicamente en su estudio, de manera que se puedan plantear modelos que permitan relacionar y definir la capacidad predictiva entre las distintas variables que se recomienda estudiar, para finalmente estructurar programas de intervención que permitan prevenir patologías psicológicas que puedan afectar el proceso de enfermedad de estos niños y adolescentes.

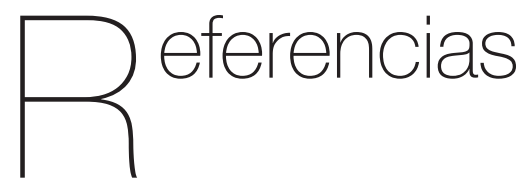

American Psychological Association. (2017). Ethical principles of psychologists and code of conduct. Recuperado de http://www. apa.org./ethics/code/ethics-code-2017.pdf

Balázs, J., Miklósi, M., Keresztény, A., Hoven, C. W., Carli, V., Wasserman, C., ... Wasserman, D. (2018). Comorbidity of physical and anxiety symptoms in adolescent: functional impairment, selfrated health and subjective well-being. International Journal of Environmental Research and Public Health, 15(8), 1-12. doi: 10.3390/ijerph15081698

Burgos, A. (2012). Propiedades psicométricas de la escala de bienestar psicológico y su estructurafactorialenuniversitarioschilenos. Psicoperspectivas. Individuo y Sociedad, 11(2), 143-163. http://doi.org/10.5027/ psicoperspectivas-Vol11-Issue2-fulltext-196

Concepción, C. (2015). Estudio sobre el bienestar psicológico y subjetivo en personas mayores y personas jóvenes y su relación con la salud. (Trabajo de Grado). 
Universidad de la Laguna, San Cristóbal de la Laguna, España.

Deledda, G., Poli, S., Giansante, M., Zamboni, M., Turazza, M. \& Gori, S. (2015). Psychological well-being, acceptance and psychological flexibility, in breast cancer patients undergoing mastectomy or lumpectomy. Annals of Oncology, 26(6), 123-131.

Díaz, D., Rodríguez, R., Blanco, A., Moreno, B., Gallardo, I., Valle, C. ... Van Dierendonck, D. (2006). Adaptación española de las Escalas de Bienestar Psicológico de Ryff. Psicothema, 18(3), 572-577.

Diener, E. (2006). Understanding Scores on the Satisfaction with Life Scale. Illinois: Psychology. University of Illinois at Urban Champaign.

Fernández, O., Muratori, M. \& Zubieta, E. (2013). Bienestar eudaemónico y soledad emocional y social. Boletín de Psicología, 108, 7-23.

García, M. A. (2002). El bienestar subjetivo. Escritos de Psicología, 6, 18-39.

International Union of Psychological Science. (2008). Declaración Universal de Principios Éticos para Psicólogos. Recuperado de https://www.ucc.ie/en/media/academic/ appliedpsychology/ethicsforms/universal_ declaration_of_ethics.pdf

Keyes, C.L.M., Shmotkin, D., \& Ryff, C.D. (2002), Optimizing well-being: The empirical encounter of two traditions. Journal of Personality and Social Psychology, 82(6), 1007-1022.

Liberalesso, A. (2002).Bienestar subjetivo en la vida adulta y la vejez: hacia una psicología positiva en América Latina. Revista Latinoamericana de Psicología, 34(1-2), 55-74.

McCullough, G., Huebner, E.S. \& Laughlin, J.E. (2000). Life events, self concept and adolescents' positive subjective well-being. Psychology in the Schools, 37(3), 281-290.

Meléndez, J. C., Tomás, J. M., \& Navarro, E. (2008). Análisis del bienestar en la vejez según la edad. Revista Española de Geriatría y Gerontología, 43(2), 90-95.

Mella, R., González, L., D'Appolonio, J., Maldonado, I., Fuenzalida, A., \& Díaz, A. (2004). Factores asociados al bienestar subjetivo en el adulto mayor. Psykhe, 13(1), 79-89.

Ministerio de Salud. (2011). 33 mil personas al año mueren de Cáncer en Colombia. [En línea] Minsalud.gov.co. Recuperado de https://www.minsalud.gov.co/Paginas/33mil-personas-al-a\%C3\%B1o-mueren-deC\%C3\%A1ncer-en-Colombia.aspx.

Ministerio de Salud. (2013). Cáncer Infantil 2014. Recuperado de https://www.minsalud.gov. co/sites/rid/Lists/BibliotecaDigital/RIDE/ VS/ED/GCFI/Presentaci\%C3\%B3n\%20 CA\%2OInfantil.pdf

Muratori, M. \& Zubieta, E. (2015). Felicidad y Bienestar Psicológico: Estudio Comparativo Entre Argentina y España Happiness and Psychological Well-Being: A Comparative Study Between Argentina and Spain. Psykhe, 24(2), 1-18.

Nearchou, F.A., Campbell, A., Duffy, K., Fehily, M., \& Ryan, H. (2017). Chronic illness stigma and well-being in youth: the mediating role of support. The European Proceedings of Social \& Behavioural Sciences, 1, 1-11. 
Novoa, M., Caycedo, C., Aguillón, M., \& Suárez, R. (2008). Calidad de vida y bienestar psicológico en pacientes adultos con enfermedad avanzada y en cuidado paliativo en Bogotá. Pensamiento Psicológico, 4(10), 177-192.

Organización Mundial de la Salud (2016). Cáncer. Recuperado de http://www.who. int/ cancer/es/

Orcasita, L.T., Mosquera, J.A., \& Carrillo, T. (2018). Autoconcepto, autoeficacia y conductas sexuales de riesgo en adolescentes. Informes Psicológicos, 18(2),141-168. http://dx.doi.org/10.18566/ infpsic.v18n2a08

Páez, D. (2008). Cuaderno de prácticas de psicología social y salud: Fichas Técnicas sobre Bienestar y Valoración Psico-Social de la Salud Mental. Recuperado de https://www.ehu.eus/ documents/1463215/1492921/Salud+200 8+Ficha+Tecnica+I++Bienestar+y+Valoraci ón+Psico-Social+de+la+Salud+Mental

Pavot, W. \& Diener, E. (2008). The Satisfaction With Life Scale and the emerging construct of life satisfaction. The Journal of Positive Psychology, 3(2), 137-152. Recuperado de http://phd.meghan-smith.com/wpcontent/uploads/2015/09/The-SWLscale-and-the-emerging-construct-of-lifesatisfaction.pdf

Pérez, C. L. (2015). El impacto del cáncer infantil en el contexto familiar desde la experiencia vital de los/as progenitores/ as (Tesis de Maestría). Universidad de La Laguna, España. Recuperado de https:// riull.ull.es

Pineda, C., Castro, J., \& Chaparro, R., (2018). Estudio psicométrico de las Escalas de
Bienestar Psicológico de Ryff en adultos jóvenes colombianos. Pensamiento Psicológico, 16(1), 45-55.

Proctor, C. (2014). Subjective well-being. En Michalos, A. (ed.), Encyclopaedia of quality of life and well-being research (pp. 64376441). New York: Springer Publishing Company.

Reina, M., Oliva, A., \& Parra, A. (2010). Percepciones de autoevaluación: Autoestima, autoeficacia y satisfacción vital en la adolescencia. Psychology, Society y Education, 2, 47-59.

Ryff, C.D., \& Keyes, C. L. M. (1995). Thestructure ofpsychologicalwell-being revisited. Journal of Personality and Social Psychology, 57, 1069-1081. Recuperado de http://dx.doi. org/10.1037//0022-3514.69.4.719

Ryff, C. D. (2017). Eudaimonic well-being, inequality, and health: Recent findings and future directions. International Review of Economics, 64(2), 159-178.

Telfer, E. (1980). Happiness. New York: St. Martin's Press.

Van Dierendonck, D. (2004). The construct validity of ryff's scales of psychological well-being and its extension with spiritual well-being. Personality and Individual Differences, 36, 629-644. Retrieved from http://dx.doi.org/10.1016/ S0191-8869(03)00122-3

Valencia, S.C. (2006). Depresión en personas diagnosticadas con cáncer. Diversitas, 2(2), 241-257. Recuperado de http://pepsic.bvsalud.org/scielo. php?script=sci_arttext\&pid=S1794 99982006000200006\&lng=pt\&tlng= es 
Vázquez, C., Duque, A., \& Hervás, G. (2013). Satisfaction with Life Scale in a Representative Sample of Spanish Adults: Validation and Normative Data. Spanish Journal of Psychology, 16(82), 1-15. Doi:10.1017/sjp.2013.82

Vinaccia, S., Riveros, F., Vera, A., Quiceno, J.M., \& Parra, N. (2019). Escala de satisfacción con la vida (SWLS): análisis de validez, confiabilidad y baremos preliminares para adultos jóvenes colombianos. Psicogente, 22(2), 1-20. Recuperado de https://revistas. unisimon.edu.co/index.php/psicogente/ article/download/3468/4161
Warner, E.L., Kent, E.E., Trevino, K.M., Parsons, H.M., Zebrack, B.J., \& Kirchhoff, A.C. (2016). Social well-being among adolescents and young adults with cancer: A systematic review. Cancer, 122(7), 102937. doi: 10.1002/cncr.29866

Žukauskienè, R. (2013). Adolescence and WellBeing. En Dordrecht (Series Ed.) Handbook of Child Well-Being: Theories, Methods and Policies in Global Perspective, (pp. 17131738) doi:10.1007/978-90-481-9063-8_67 